\title{
Fading of the X-Ray Afterglow of Neutron Star Merger GW170817/GRB 170817A at 260 Days
}

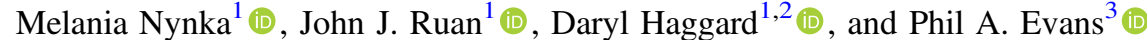 \\ ${ }^{1}$ McGill Space Institute and Department of Physics, McGill University, 3600 rue University, Montreal, Quebec, H3A 2T8, Canada; melania.nynka@mcgill.ca \\ ${ }^{2}$ CIFAR Azrieli Global Scholar, Gravity \& the Extreme Universe Program, Canadian Institute for Advanced Research, \\ 661 University Avenue, Suite 505, Toronto, ON M5G 1M1, Canada \\ ${ }^{3}$ Leicester Institute for Space and Earth Observation and Department of Physics \& Astronomy, University of Leicester, University Road, Leicester, LE1 7RH, UK \\ Received 2018 May 10; revised 2018 July 5; accepted 2018 July 12; published 2018 July 31
}

\begin{abstract}
The multi-wavelength electromagnetic afterglow from the binary neutron star merger GW170817/GRB 170817A has displayed long-term power-law brightening, and has presented challenges to post-merger models of the nonthermal emission. The most recent radio observations up to 200 days post-merger suggest that the afterglow has finally peaked and may now be fading, but fading has not been confirmed in the X-rays. We present new, deep Chandra observations of GW170817/GRB 170817A at 260 days post-merger that reveal an X-ray flux of $F_{0.3-8 \mathrm{keV}}=1.1 \times 10^{-14} \mathrm{erg} \mathrm{s}^{-1} \mathrm{~cm}^{-2}$, and confirm that the X-ray light curve is now also fading. Through rigorous comparisons with previous Chandra observations of GW170817/GRB 170817A, X-ray fading is detected between 160 and 260 days post-merger at a $4.4 \sigma$ significance on the basis of the X-ray data alone. We further constrain the X-ray photon index to steepen by $<0.5$ at $3.1 \sigma$ significance during this period, which disfavors the passing of the synchrotron cooling frequency through the X-ray band as the cause of the observed fading. These observations remain consistent with optically thin synchrotron afterglow emission. If this afterglow emission arises from a quasi-spherical mildly relativistic outflow, the X-ray fading suggests that the outflow is now decelerating. Alternatively, if this afterglow arises from a successful off-axis structured jet, the X-ray fading suggests that emission from the jet core has already entered the line of sight.
\end{abstract}

Key words: galaxies: individual (NGC 4993) - gamma-ray burst: individual (GRB 170817A) - gravitational waves - stars: neutron - X-rays: binaries

\section{Introduction}

The discovery of multi-wavelength electromagnetic emission from the binary neutron star (NS) merger GW170817 heralded the dawn of multi-messenger gravitational wave astronomy (e.g., Abbott et al. 2017a, 2017b; Coulter et al. 2017; Evans et al. 2017; Goldstein et al. 2017; Hallinan et al. 2017; SoaresSantos et al. 2017; Troja et al. 2017; Valenti et al. 2017). The short gamma-ray burst (sGRB) GRB 170817A associated with this gravitational wave event confirmed that the progenitors of at least some sGRBs can be binary NS mergers (BNS; Abbott et al. 2017c; Goldstein et al. 2017; Savchenko et al. 2017). Furthermore, the optical and infrared transient associated with GW170817 confirmed that the ejecta from binary NS mergers are the sites of $r$-process nucleosythesis, which is in broad agreement with predictions from kilonova models (Arcavi et al. 2017; Cowperthwaite et al. 2017; Drout et al. 2017; Kasen et al. 2017; Kasliwal et al. 2017; McCully et al. 2017; Pian et al. 2017; Shappee et al. 2017; Smartt et al. 2017). However, nearly nine months after its first detection, the postmerger evolution of this binary NS merger remains unclear, and the non-thermal electromagnetic emission is still rapidly evolving.

The early X-ray and radio light curves of GW170817/ GRB 170817A are unlike any other sGRB previously observed. Early X-ray observations of the electromagnetic counterpart resulted in only upper limits on the X-ray flux (Evans et al. 2017; Margutti et al. 2017). An initial Chandra X-ray detection was first made at $\sim 9$ days post-merger (Troja et al. 2017), and was confirmed in additional Chandra observations at $\sim 15$ days (Haggard et al. 2017). Similarly, early radio observations resulted in non-detections (Alexander et al. 2017) until a first detection at $\sim 16$ days post-merger (Hallinan et al. 2017). This delayed rise of the X-ray and radio emission is not observed in classical sGRB afterglows, which display monotonic fading over timescales of days (Fong et al. 2017). The X-ray and radio emission of GRB 170817A was instead initially suggested to be consistent with models of a synchrotron afterglow from a simple top-hat sGRB jet observed off-axis, or a simple mildly relativistic cocoon blast wave (e.g., Alexander et al. 2017; Haggard et al. 2017; Hallinan et al. 2017; Margutti et al. 2017; Troja et al. 2017).

Continued long-term X-ray and radio monitoring of GRB 170817A has presented new challenges to post-merger models. The sky proximity of GRB 170817 A to the Sun prevented $\mathrm{X}$-ray monitoring between $\sim 16$ and $\sim 109$ days post-merger. Meanwhile, continued radio monitoring revealed that GRB $170817 \mathrm{~A}$ continued to slowly brighten following a $t^{0.8}$ power law over time up to $\sim 107$ days post-merger (Mooley et al. 2018b). The slow long-term brightening of the afterglow emission now disfavors models of top-hat off-axis jets or simple cocoon blast waves, which predict steeper power-law brightening than has been observed. This conclusion was strengthened by Chandra X-ray observations at $\sim 109$ days (immediately after Sun restrictions were lifted), which revealed that the X-ray emission brightened at a similar rate (Ruan et al. 2018). More recent $X$-ray observations at $\sim 160$ days suggested that the afterglow light curve may be peaking (D'Avanzo et al. 2018; Margutti et al. 2018), and radio observations up to $\sim 200$ days indicated that the afterglow may have begun fading (Dobie et al. 2018). 
It is still unclear how the synchrotron afterglow emission arises during the post-merger evolution of GRB 170817A. The consistency between the observed radio spectral index, X-ray spectral index, and broadband radio-to-X-ray spectral index (all $\alpha \sim 0.6)$ conclusively shows that the spectral energy distribution of GRB 170817A is a single $F_{\nu} \sim \nu^{-0.6}$ power law that spans from X-ray through radio frequencies (e.g., Margutti et al. 2018; Mooley et al. 2018b; Ruan et al. 2018). This spectrum is consistent with optically thin synchrotron emission from a trans-relativistic shock with a Lorentz factor of $\Gamma \approx 3-10$ (Lyman et al. 2018; Margutti et al. 2018). The constant slope of the synchrotron power-law spectrum between radio and X-ray frequencies up to 160 days implies that the synchrotron self-absorption frequency is below $\sim 1 \mathrm{GHz}$, while the synchrotron cooling frequency is above $\sim 1 \mathrm{keV}$.

Currently, the most promising models for the synchrotron afterglow invoke interactions between the relativistic jet and the merger ejecta, and can be crudely divided into (1) quasispherical, mildly relativistic outflows and (2) off-axis angularly structured jets. In outflow models, either dynamical ejecta or a cocoon shocked by a choked jet drives a mildly relativistic afterglow shock into the surrounding interstellar medium (e.g., Gottlieb et al. 2018b, 2018a; Lazzati et al. 2017b; Nakar \& Piran 2017; Mooley et al. 2018b). This shock can be approximated as a quasi-spherical blast wave, which will accelerate electrons that produce synchrotron emission in the shock-generated magnetic field. If the outflow is radially stratified, such that the majority of the kinetic energy is in the lower-velocity material, the blast wave experiences a continuous injection of energy in its coasting phase. The resulting afterglow emission will thus slowly brighten, as seen in the observations, particularly in comparison to simple nonstratified blast waves (Mooley et al. 2018b). In contrast, for structured jet models, the relativistic jet successfully breaks out of the ejecta, but is viewed off-axis (e.g., Lamb \& Kobayashi 2017; Lazzati et al. 2017a; Kathirgamaraju et al. 2018; Lyman et al. 2018; Margutti et al. 2018). The key feature of structured jet models is that the jet has an angular structure, where the Lorentz factor, $\Gamma$, of the jet decreases gradually as a function of angle from the jet axis, possibly due to interaction with kilonova ejecta (Lazzati et al. 2017a; Xie et al. 2018). When viewed off-axis, the observed synchrotron afterglow is dominated by jet material with increasingly larger initial $\Gamma$ over time, as the jet decelerates and material that was more initially relativistic enters the line of sight. This causes the structured jet afterglow to brighten slowly, similar to the observed X-ray and radio light curves, especially in comparison with jets with a top-hat distribution in $\Gamma$. Current multi-wavelength observations of the afterglow of GRB 170817A during its brightening cannot distinguish between structured jets and outflows (e.g., Nakar et al. 2018; Nakar \& Piran 2018; Margutti et al. 2018), although some models of these two scenarios have predicted divergent afterglow light-curve characteristics during the fading after its light-curve peak (Lamb et al. 2018; Lyman et al. 2018; Troja et al. 2018). Furthermore, recent VLBI imaging suggest that the radio morphology of GRB 170817A supports a successful off-axis angularly structured jet (Mooley et al. 2018a).

Breaks in the afterglow light curve-such as a light-curve peak or a change in the light-curve power-law slope-place important constraints on models for the origin of its nonthermal emission. For an off-axis structured jet, a peak in the afterglow light curve will occur when emission from the jet core enters the line of sight (e.g., van Eerten et al. 2010; Lyman et al. 2018). This geometric jet break is expected to be achromatic (occurring at all wavelengths simultaneously), and the timing of the break jointly constrains parameters such as the jet opening angle and the jet axis angle from the line of sight. For a quasi-spherical outflow with radially stratified kinetic energy, a peak in the afterglow light curve will occur at the onset of deceleration of the slowest-moving material in the outflow shock (e.g., Nakar \& Piran 2017). This light-curve break is also expected to be achromatic, and the timing of the break jointly constrains parameters such as the kinetic energy structure and Lorentz factor of the outflow. However, model interpretations and constraints based on an observed light-curve break must first rule out other possible origins for the break.

The passing of the synchrotron cooling frequency, $\nu_{\mathrm{c}}$, through the X-ray band can also cause a beak in the X-ray light curve. For shock-accelerated relativistic electrons in the slowcooling regime with a power-law distribution of energies, $\nu_{\mathrm{c}}$ corresponds to the frequency above which electrons have now radiatively cooled. Because synchrotron radiative losses scale with particle energy, $\nu_{\mathrm{c}}$ will decrease in frequency over time, crossing the X-ray band first before affecting the radio. The resulting characteristic steepening of the power-law spectrum across the cooling frequency of $\Delta \Gamma_{X}=0.5$ (where $\Gamma_{X}$ is the $\mathrm{X}$-ray photon index) causes a chromatic light-curve break in which the X-ray light curve will fade before the radio. Observations up to 160 days post-merger show that the afterglow of GRB 170817A continues to display a single power-law spectral energy distribution that spans from X-ray to radio frequencies (e.g., Lyman et al. 2018; Margutti et al. 2018; Ruan et al. 2018). This implies that $\nu_{\mathrm{c}}$ was still above X-ray frequencies at that time, though it will eventually pass through the Chandra band.

The exact timing of when $\nu_{\mathrm{c}}$ is observed to cross the X-ray band is model dependent, and sensitive to model parameters. For example, in outflow models, $\nu_{\mathrm{c}}(t)$ is most strongly dependent on the outflow velocity (Mooley et al. 2018b). The lower-velocity dynamical ejecta outflow model of Hotokezaka et al. (2018) predicts that the $\nu_{\mathrm{c}}$ will cross the X-ray band on timescales of a few months to a year post-merger, while higher-velocity cocoon outflow models predict longer timescales of several years (Mooley et al. 2018b). Simulations of structured jets have predicted that $\nu_{\mathrm{c}}$ will stay above X-ray frequencies for several years (Lazzati et al. 2017a; Margutti et al. 2018). Thus, a potential detection of a synchrotron cooling break through the $\mathrm{X}$-rays in the near future would support the dynamical ejecta outflow model for the afterglow, while a non-detection would support either cocoon outflow models or structured jets. In any case, if a peak or break in the X-ray light curve is detected, interpretations for the origin of the break should first rule out synchrotron cooling as the cause of the break.

Although radio observations up to 200 days post-merger hinted that the afterglow of GRB 170817A may have begun fading (Dobie et al. 2018), it has been unclear if this fading is also observed in X-rays. A recent Chandra detection at 160 days suggest that the X-rays light curve is peaking (Margutti et al. 2018), but fading has yet to be confirmed. In this Letter, we present new, deep Chandra X-ray observations of GRB 170817A at 260 days post-merger, the first since the last Chandra observation at 160 days. Our analysis of these new data and comparisons with previous Chandra observations 


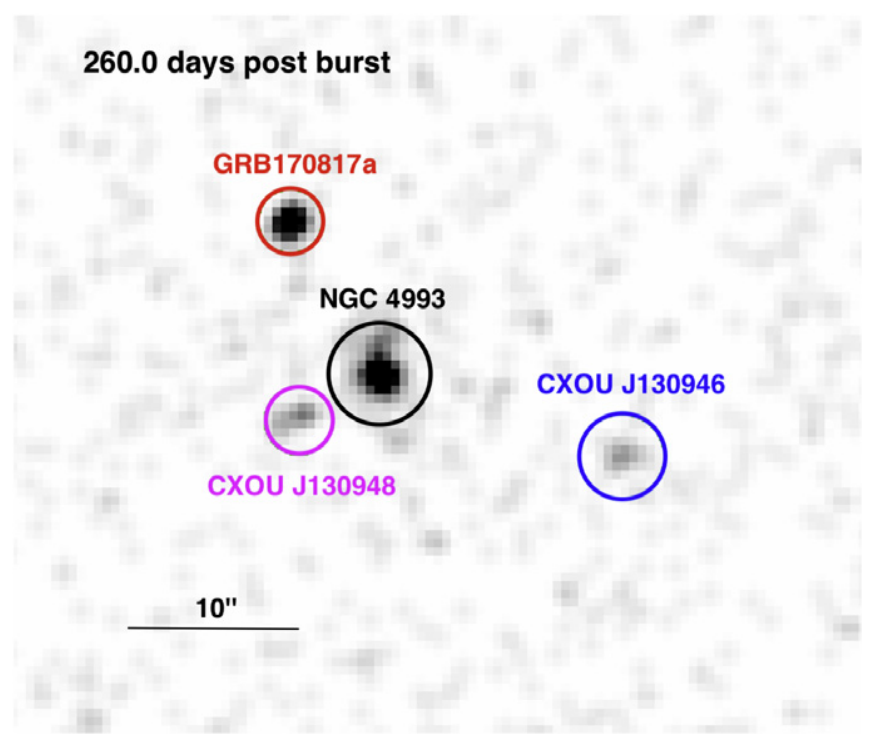

Figure 1. Latest Chandra $0.5-8.0 \mathrm{keV}$ X-ray image of GRB $170817 \mathrm{~A}$ at 260 days post-merger, in a $96.8 \mathrm{ks}$ total observation. The X-ray afterglow of GW170817/GRB 170817A is still clearly detected, along with X-ray emission from the host galaxy (NGC 4993) and two other previously detected sources in the field. This image is shown on a linear scale, and has been smoothed with a 2 pixel Gaussian kernel.

reveal that the X-ray emission is now also fading (see also Alexander et al. 2018). Furthermore, we do not detect the characteristic steepening of the X-ray photon index expected from a synchrotron cooling break, thus disfavoring this possibility for the origin of the X-ray fading. In Section 2, we describe our new data and analysis procedure. In Section 3, we compare our newest observations with previous Chandra observations of GRB 170817, to test for fading and changes in the X-ray photon index. We briefly conclude in Section 4.

\section{X-Ray Observations and Analysis}

\subsection{New Chandra Observations at 260 days Post-merger}

We have obtained new, deep Chandra X-ray observations of GRB 170817A via a Director's Discretionary Time allocation (PI: Wilkes, Program Number 19408644). Two exposures of GRB 170817A were collected: (1) a $50.8 \mathrm{ks}$ exposure (ObsID 21080) beginning at 2018 May 3.08 UT, approximately 259 days post-merger, and (2) a $46.0 \mathrm{ks}$ exposure (ObsID 21090) beginning at 2018 May $4.08 \mathrm{UT}$, approximately 261 days postmerger. These two exposures where acquired using the ACISS3 chip in VFAINT mode. We use the CIAO v.4.10 software (CALDB v4.7.8; Fruscione et al. 2006) to reduce and analyze these Chandra data. We first use CIAO's repro script to reprocess all level-2 events files and apply the latest calibrations. To produce a deep X-ray image, we co-add the two exposures into a single $96.8 \mathrm{ks}$ exposure at 260.0 days post-merger. The X-ray emission from GRB 170817A is not expected to vary significantly over the2-day timescales covered by these two exposures. The co-added 0.5-8 Chandra keV image of GRB 170817A at 260 days is shown in Figure 1. $\mathrm{X}$-ray emission is still clearly detected at the position of GRB 170817A in these latest data, as well as at the positions of the three other nearby X-ray sources observed previously: CXOU J130948, CXOU 130946, and the host galaxy NGC 4993.
We determine the centroid position of GRB 170817A in each of the two individual $0.5-7 \mathrm{keV}$ images using the wavdetect detection algorithm. We then extract X-ray spectra using regions with radii of 1 !"97. This extraction radius corresponds to a $\sim 90 \%$ encircled energy fraction near the Chandra on-axis position. To determine the background, we use a large region from the same chip that does not overlap the extraction region of the detected sources.

We extract X-ray spectral and response files from the two individual observations of GRB 170817A using the specextract tool, and co-add them into a single spectrum using combinespectra to improve statistics. We use XSPEC v12.9.0 (Arnaud 1996) to fit the co-added spectra, with atomic cross sections from Verner et al. (1996) and abundances from Wilms et al. (2000). For each source, we assume an absorbed power-law spectral model tbabs*powerlaw with fixed absorption $N_{\mathrm{H}}=7.5 \times 10^{20} \mathrm{~cm}^{-2}$. A distance of $42.5 \mathrm{Mpc}$ is adopted throughout, obtained from the host galaxy NGC $4993\left(z=0.0098 D_{L}=42.5 \pm 0.3\right.$ da Costa et al. 1988).

In this co-added $96.8 \mathrm{ks}$ observation at 260 days, we measure GRB 170817's source count rate to be $7.9 \times 10^{-4}$ counts $^{-1}(0.5-8 \mathrm{keV})$, a factor of $\sim 2$ fainter than in the $104.9 \mathrm{ks}$ Chandra observation at 160 days. This count rate at 260 days corresponds to an absorbed flux of $F_{0.3-8 \mathrm{keV}}=10.9 \times 10^{-15} \mathrm{erg} \mathrm{s}^{-1} \mathrm{~cm}^{-2}$, and an unabsorbed luminosity of $L_{0.3-10 \mathrm{keV}}=2.96 \times 10^{39} \mathrm{erg} \mathrm{s}^{-1}$. The extracted $\mathrm{X}$-ray spectrum of GRB 170817A is shown in Figure 3 (right panel) along with the best-fit spectral model. The spectrum is well described by the assumed absorbed power-law model, with $\chi_{\nu}^{2}=0.89$. Table 1 lists the best-fit power-law photon index and absorbed $0.3-8 \mathrm{keV}$ flux, in comparison to previous observations.

\subsection{Uniform Re-reduction of Previous Chandra Observations}

To enable a consistent and uniform comparison of the latest Chandra observations of GRB 170817A with previous observations, we systematically re-reduce and analyze all currently available Chandra data using the exact same procedure as in Section 2.1. For these 13 observations (listed in Table 1), we group observations that are close in time into coadded observations at 2.3, 9.2, 15.6, 109.2, and 159.7 days. The Chandra data from the observation at 2.3 days are not currently publicly available, so we rescale the $5 \sigma$ upper limit on the flux from Margutti et al. (2017) to an absorbed flux in the 0.3-8 keV band. An updated Chandra light curve of GRB 170817A using these fluxes is shown in Figure 2, and the spectral fits to the 109 and 160 days observations are shown in Figure 3 (left panel). The resultant count rates, fluxes, and model parameters are consistent with previously reported values. In Section 3, we use this uniformly reduced data set to statistically test whether the X-ray emission from GRB 170817A has faded in the latest observations, and whether the $\mathrm{X}$-ray photon index has steepened as expected for a synchrotron cooling break.

\section{Comparisons with Previous Data}

\subsection{Did the X-Ray Emission Fade?}

We test whether the latest Chandra observations at 260 days display statistically significant fading in comparison with the previous observation at 160 days. Comparison of the observed 
Table 1

Chandra X-ray Properties of GW170817/GRB 170817A

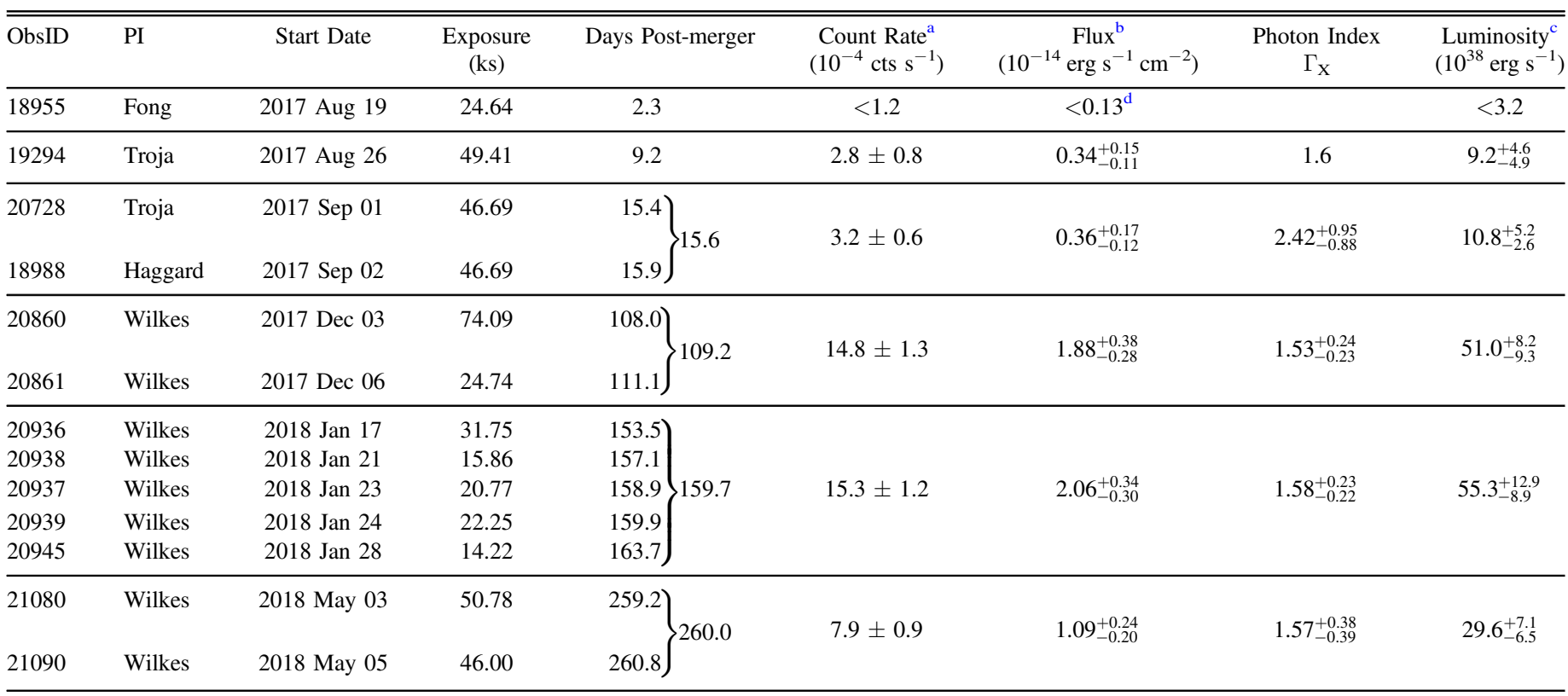

Notes. All reported uncertainties represent $90 \%$ confidence intervals. The neutral hydrogen absorption was frozen to $N_{\mathrm{H}}=7.5 \times 10^{20} \mathrm{~cm}^{-2}$ for all spectral fits, based on NGC 4993's $A_{\mathrm{V}}=0.338$ (Schlafly \& Finkbeiner 2011).

a $0.5-8 \mathrm{keV}$ absorbed.

${ }^{\mathrm{b}} 0.3-8 \mathrm{keV}$ absorbed.

c $0.3-10 \mathrm{keV}$ unabsorbed, assuming a luminosity distance of $42.5 \mathrm{Mpc}$.

${ }^{\mathrm{d}}$ Rescaled from Margutti et al. (2017).

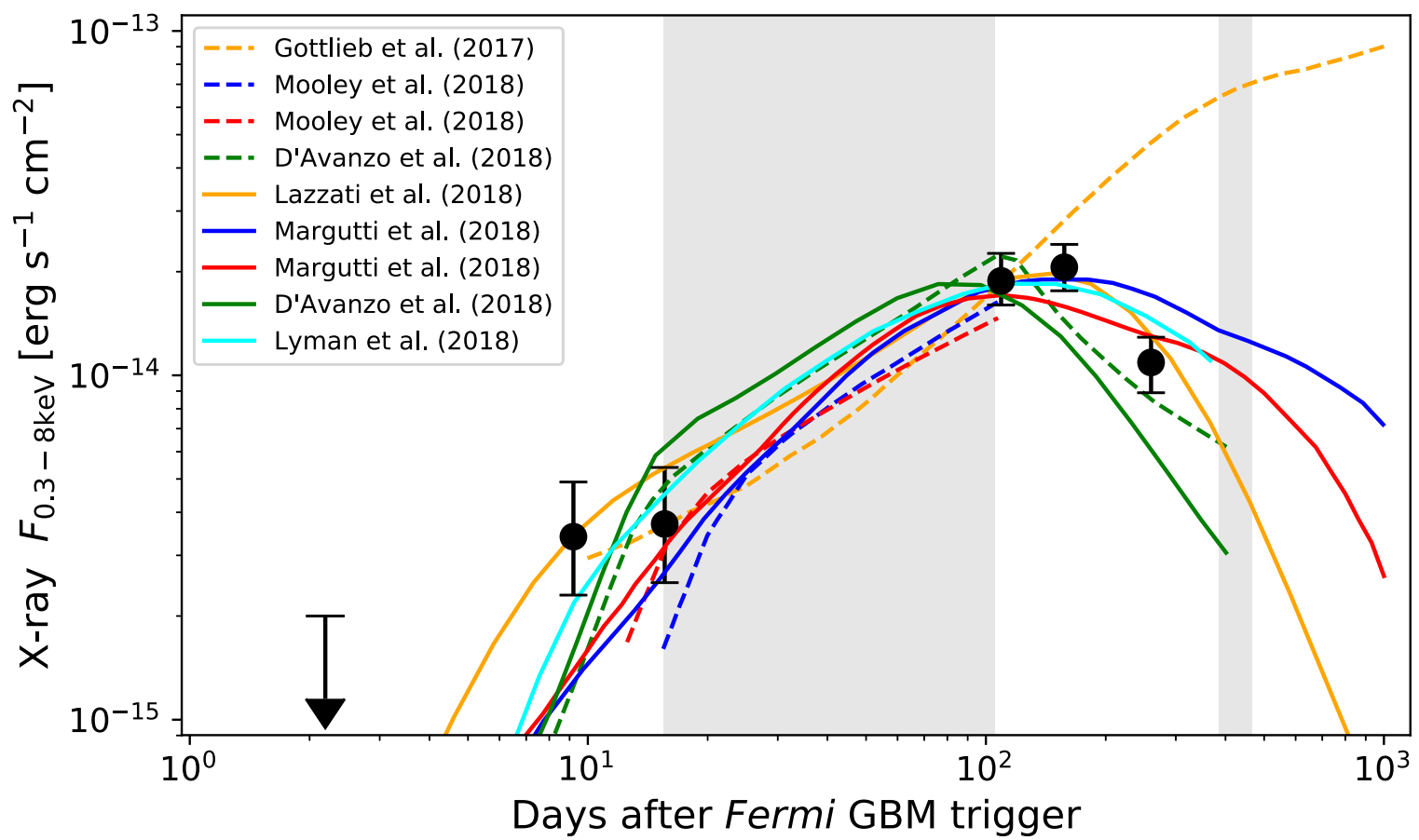

Figure 2. Chandra X-ray light curve of GW170817/GRB 170817A (black points), including the new observations at 260 days post-merger. The fluxes for all previous Chandra observations are from a uniform re-reduction of the data (see Section 2.2). X-ray light-curve predictions from a selection of quasi-spherical outflow models (dashed lines) and structured jet models (solid lines) are also shown for comparison. We note that these models are fitted or matched to various combinations of previous X-ray/radio observations, and have flexible parameters that can produce a wide range of light-curve peak times. Thus, the fading of the X-ray light curve revealed by our latest data at 260 days does not necessarily rule out any of the models shown. The gray shaded regions are time-spans over which Chandra observations are not possible, due to Sun constraints. All uncertainties shown are $90 \%$ confidence level, and upper limits are $5 \sigma$ confidence. 

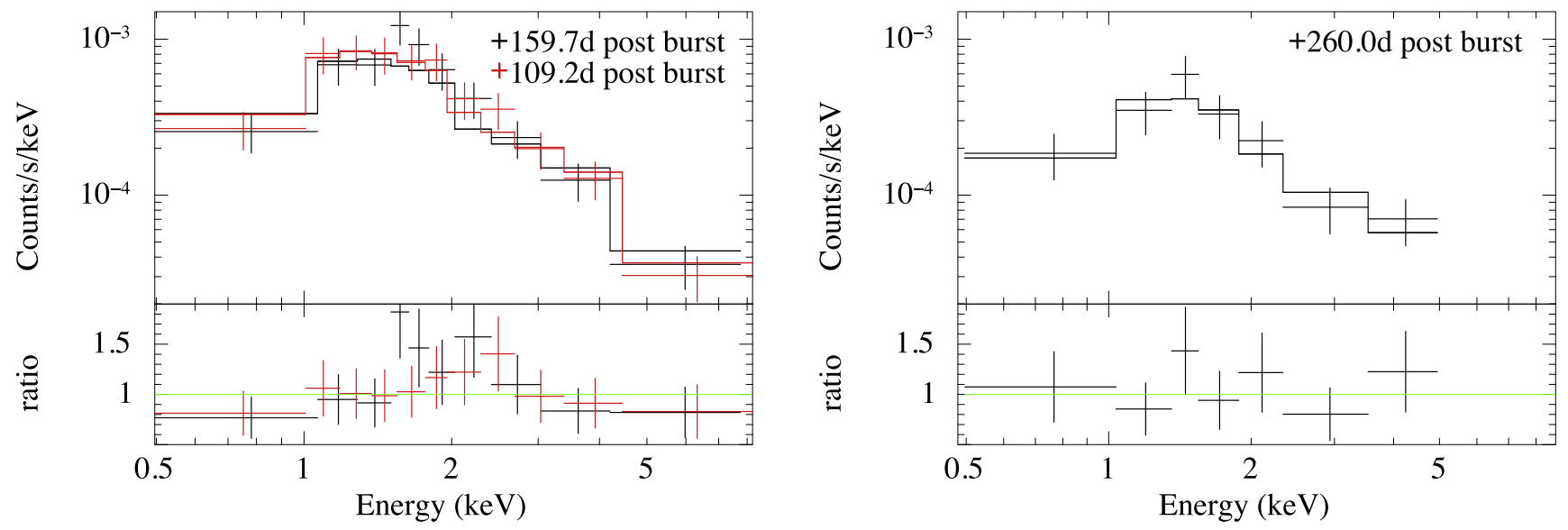

Figure 3. Chandra X-ray spectra of GW170817/GRB 170817A in the latest 96.8 ks observation at 260 days (right panel), compared with spectra from the previous $\sim 100$ ks observations at 109 and 160 days (left panel). The best-fit absorbed power-law spectral models (solid lines) are jointly fit to their respective data sets: at 109 days $\Gamma_{\mathrm{X}}=1.53_{-0.23}^{+0.24}$, at 160 days $\Gamma_{\mathrm{X}}=1.58_{-0.22}^{+0.23}$, and at 260 days $\Gamma_{\mathrm{X}}=1.57_{-0.39}^{+0.38}$. The neutral hydrogen absorption column is fixed to $N_{\mathrm{H}}=7.5 \times 10^{20} \mathrm{~cm}^{-2}($ see Table 1 and Section 2 for details). The lack of evolution in the spectral index disfavors the passing of the synchrotron cooling frequency through the X-ray band over the time interval probed to date.
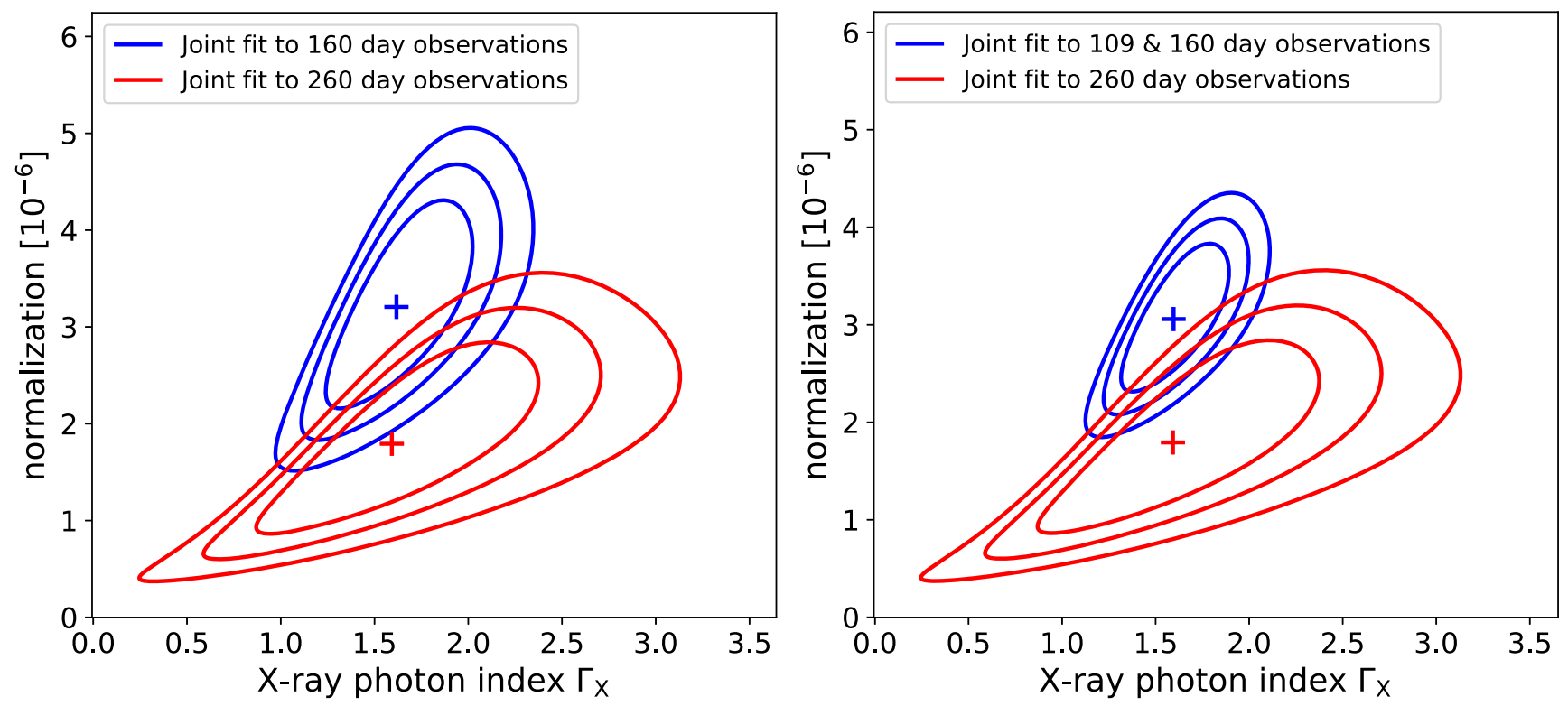

Figure 4. Left: comparison of the joint constraints on the X-ray photon index $\Gamma_{\mathrm{X}}$ and the flux normalization of the power-law X-ray spectrum of GRB 170817A, between the 160 days Chandra observations (blue contours) and the new 260 days observations (red contours). The contours show the $1 \sigma$, $90 \%$, and $2 \sigma$ confidence levels, and the best-fitting parameters are indicated with a cross. We constrain the X-ray emission to be fading between these two epochs (i.e., with normalization decreasing) at a $4.4 \sigma$ significance, by marginalizing over $\Gamma_{\mathrm{X}}$ (see Section 3.1). Right panel: similar joint constraints, comparing between the jointly fitted 109 and 160 days data with the new 260 days observations. We constrain the X-ray photon index to steepen by $<0.5$ at a $3.1 \sigma$ significance by marginalizing over the normalization parameter, thus disfavoring a synchrotron cooling break as the cause of the fading (see Section 3.2).

$2-8 \mathrm{keV}$ count rates at 160 and 260 days from Table 1 shows a decrease with $4.9 \sigma$ significance. However, this simplistic approach is useful only with the assumption that the spectral shape remains identical between the observations. Similarly, the X-ray fluxes also cannot be directly compared to test for fading for two reasons. First, there is a non-zero covariance between the power-law normalization $N$ and slope $\Gamma_{\mathrm{X}}$ parameters in the spectral power-law model $N \times E^{-\Gamma_{\mathrm{X}}}$ (Arnaud 1996). The normalization is defined as the intensity $\left(\mathrm{ph} \mathrm{s}^{-1} \mathrm{~cm}^{-2} \mathrm{keV}^{-1}\right.$ ) of the source at $1 \mathrm{keV}$. Integrated flux (with units of $\mathrm{erg} \mathrm{s}^{-1} \mathrm{~cm}^{-2}$ is dependent on both these parameters, thus a change in the measured fluxes in Table 1 could be due to a change in $N$ and/or $\Gamma_{\mathrm{X}}$. Second, the uncertainties on both $N$ and $\Gamma_{\mathrm{X}}$ are non-Gaussian, and may have long tails that produce a large apparent change in the measured flux. Our approach is to marginalize the joint likelihood $\mathcal{L}\left(N, \Gamma_{\mathrm{X}}\right)$ at these two epochs over $\Gamma_{\mathrm{X}}$, and then compare the marginalized likelihood $\mathcal{L}(N)$ to test for a decrease in the power-law normalization between 160 and 260 days.

We first produce a joint likelihood $\mathcal{L}\left(N, \Gamma_{\mathrm{X}}\right)$ for both the 160 and 260 days observations. We use XSPEC to generate a $1000 \times 1000$ grid of $\chi^{2}$ as a function of both $\Gamma_{\mathrm{X}}$ and $N$, shown in Figure 4 (left panel). We then use a $\exp \left(-\chi^{2} / 2\right)$ likelihood function to compute the joint likelihood $\mathcal{L}\left(N, \Gamma_{\mathrm{X}}\right)$. We marginalize over $\Gamma_{\mathrm{X}}$ to produce a marginalized $\mathcal{L}(N)$, for both the 160 and 260 days observations.

To test for a decrease in the power-law normalization $N$ (i.e., fading of the X-ray spectrum), we normalize the $\mathcal{L}(N)$ to produce a probability density function $p(N)$ for the 160 and 260 days observations. We then resample the two probability 
density functions to generate a distribution of $\Delta N$ between the two epochs. To perform the resampling of $p(N)$, we calculate the cumulative distribution function and use the inverse transform resampling method. We generate $10^{7}$ resampled values for $N$ at both 160 and 260 days, and produce a distribution of $\Delta N$. Based on this $\Delta N$ distribution, we constrain the power-law spectrum normalization to be decreasing (i.e., $\Delta N<0$ ) with $4.4 \sigma$ significance. Thus, our test shows that the X-ray emission from GRB 170817A displays statistically significant fading between 160 and 260 days post-merger. Our conclusion based solely on X-ray data is consistent with alternative approaches to test for fading in the afterglow of GRB 170817A based on joint fitting of X-ray, radio, and optical light curves (Alexander et al. 2018).

\subsection{Did the X-Ray Photon Index Steepen?}

The statistically significant fading of the X-ray emission from GRB 170817A revealed in the latest Chandra observations at 260 days can occur if the synchrotron cooling frequency $\nu_{\mathrm{c}}$ has recently passed through the Chandra band. In this scenario, the X-ray photon index $\Gamma_{\mathrm{X}}$ would be observed to steepen by a characteristic $\Delta \Gamma_{X}=0.5$, and the X-ray light curve would fade faster than the radio until $\nu_{\mathrm{c}}$ later passes through radio frequencies. Thus, we test for synchrotron cooling as the origin of the X-ray light-curve peak, by comparing the observed change in $\Gamma_{\mathrm{X}}$ to the characteristic steepening of $\Delta \Gamma_{\mathrm{X}}=0.5$ expected from synchrotron cooling.

Similar to our test for X-ray fading in Section 3.1, a test for changes in $\Gamma_{X}$ also should not be based on a direct comparison of the fitted $\Gamma_{X}$ between two observations in Table 1. We thus follow a similar procedure as in Section 3.1 to test for changes in $\Gamma_{\mathrm{X}}$, but now marginalizing $\mathcal{L}\left(N, \Gamma_{\mathrm{X}}\right)$ over the normalization $N$. Since X-ray fading was not observed between 109 and 160 days, we jointly fit the observations between these two epochs (constraints are shown in right panel of Figure 4, and a best-fit spectral model is shown in left panel of Figure 3), and compare this joint fit with the newest observations at 260 days. Based on the distribution in $\Delta \Gamma_{X}$ between these two data sets, we constrain $\Gamma_{X}$ to steepen by $<0.5$ at a $3.1 \sigma$ significance. Thus, our test disfavors the characteristic steepening of the power-law X-ray spectrum from a synchrotron cooling break as the cause of the X-ray fading between 160 and 260 days postmerger.

\subsection{A Best-available Constraint on the X-Ray Photon Index}

We use our entire uniformly reduced Chandra data set from Section 2.2 to derive the best constraints to date for $\Gamma_{X}$. Because the X-ray flux of GRB 170817A changes over time, we cannot directly co-add all the observations, and instead we use the co-added data from the groups of observations at 9.2, $15.6,109.2,159.7$, and 260.0 days to jointly fit an absorbed power-law model. The power-law photon index $\Gamma_{\mathrm{X}}$ is tied between observations and the normalization left free. In our tests, the observations at 9.2 and 15.6 days do not improve the spectral fit, due to low count rates. We thus only use the observations at 109.2, 159.7, and 260.0 days, and derive a jointly fitted $\Gamma_{X}=1.56_{-0.15}^{+0.14}$. This is the best constraint on $\Gamma_{X}$ to date, and it is highly consistent with the current best constraints on both the radio spectral index of $\alpha_{\mathrm{R}}=0.61 \pm 0.05$ (where $\alpha=\Gamma-1$ ) (Mooley et al. 2018b), and the broadband X-rayto-radio spectral index of $\alpha_{\mathrm{XR}}=0.585 \pm 0.005$ (Margutti et al. 2018). The consistency of the X-ray photon index with the radio spectral index further supports a synchrotron powerlaw spectrum that spans from X-ray to radio frequencies.

\section{Conclusion}

We present new, deep Chandra observations of GW170817/ GRB 170817A at 260 days post-merger. These are the first X-ray observations since 160 days, which had suggested that the brightening afterglow light curve may be reaching a peak. Our analysis of the new observations reveals that GRB $170817 \mathrm{~A}$ is indeed now fading in X-rays. We compare the X-ray properties of GRB 170817A with previous Chandra observations using a uniform re-reduction of all available data. We show that the fading of the X-ray power-law spectrum is detected at a $4.4 \sigma$ significance, based on the X-ray data alone. Furthermore, we detect no change in the X-ray photon index $\Gamma_{\mathrm{X}}$, and constrain $\Gamma_{\mathrm{X}}$ to steepen by $<0.5$ at $3.1 \sigma$ significance. This disfavors a synchrotron cooling break as the cause of the $\mathrm{X}$-ray fading, which would instead result in a characteristic steeping in $\Gamma_{X}$ of 0.5 .

The X-ray fading of GRB 170817A remains consistent with current post-merger models for the synchrotron afterglow. By jointly fitting all available Chandra data to date, we derive a best-available measurement of the X-ray photon index of $\Gamma_{\mathrm{X}}=1.56_{-0.15}^{+0.14}$. The consistency of $\Gamma_{\mathrm{X}}$ with the radio spectral index implies that the afterglow spectrum remains a single power law spanning from radio-to-X-ray frequencies. For quasi-spherical mildly relativistic outflow models of the afterglow emission, the fading at radio through X-ray frequencies implies that the outflow is now in a decelerating phase. For angularly structured off-axis jets, the fading implies that emission from core of the jet has already entered the line of sight. Both Lyman et al. (2018) and Troja et al. (2018) suggest that the post-break light-curve characteristics of GRB 170817A can discriminate between the structured jet and quasi-spherical outflow models. To this end, we urge that continued X-ray monitoring of GRB 170817A be avidly pursued.

Looking forward, future detections of electromagnetic counterparts to LIGO-Virgo gravitational wave sources will ideally have long, multi-wavelength light curves. Absent this ideal, our analysis indicates that deep X-ray observations alone are sufficient to track the post-merger evolution of this binary NS merger, and monitor the progression of both light-curve breaks and potential spectral breaks as the outflow/jet evolves. If this finding is supported by future detections, it could provide an important constraint on X-ray mission design and electromagnetic follow-up strategies.

The authors thank Belinda Wilkes and the Chandra scheduling, data processing, and archive teams for making these observations possible. This work was supported by Chandra Award Number GO7-18033X, issued by the Chandra $\mathrm{X}$-ray Observatory Center, which is operated by the Smithsonian Astrophysical Observatory for and on behalf of the National Aeronautics Space Administration (NASA) under contract NAS8-03060. J.J.R., M.N., and D.H. acknowledge support from a Natural Sciences and Engineering Research Council of Canada (NSERC) Discovery grant and a Fonds de recherche du Québec-Nature et Technologies (FRQNT) Nouveaux Chercheurs grant. J.J.R. and M.N. acknowledge funding from the McGill Trottier Chair in Astrophysics and Cosmology. D.H. acknowledges support from the Canadian 
Institute for Advanced Research (CIFAR). P.A.E. acknowledges UKSA support.

Facility: $C X O$.

Software: Matplotlib (Hunter 2007).

\section{ORCID iDs}

Melania Nynka @i https://orcid.org/0000-0002-3310-1946

John J. Ruan (1) https://orcid.org/0000-0001-8665-5523

Daryl Haggard (ํ) https://orcid.org/0000-0001-6803-2138

Phil A. Evans iㅏ https://orcid.org/0000-0002-8465-3353

\section{References}

Abbott, B. P., Abbott, R., Abbott, T. D., et al. 2017a, PhRvL, 119, 161101 Abbott, B. P., Abbott, R., Abbott, T. D., et al. 2017b, ApJL, 848, L12 Abbott, B. P., Abbott, R., Abbott, T. D., et al. 2017c, ApJL, 848, L13

Alexander, K. D., Berger, E., Fong, W., et al. 2017, ApJL, 848, L21

Alexander, K. D., Margutti, R., Blanchard, P. K., et al. 2018, arXiv:1805. 02870

Arcavi, I., Hosseinzadeh, G., Howell, D. A., et al. 2017, Natur, 551, 64 Arnaud, K. A. 1996, adass V, 101, 17

Coulter, D. A., Foley, R. J., Kilpatrick, C. D., et al. 2017, Sci, 358, 155 Cowperthwaite, P. S., Berger, E., Villar, V. A., et al. 2017, ApJL, 848, L17

da Costa, L. N., Pellegrini, P. S., Sargent, W. L. W., et al. 1988, ApJ, 327, 544 D'Avanzo, P., Campana, S., Ghisellini, G., et al. 2018, A\&A, 613, L1 Dobie, D., Kaplan, D. L., Murphy, T., et al. 2018, ApJL, 858, L15 Drout, M. R., Piro, A. L., Shappee, B. J., et al. 2017, Sci, 358, 1570 Evans, P. A., Cenko, S. B., Kennea, J. A., et al. 2017, Sci, 358, 1565 Fong, W., Berger, E., Blanchard, P. K., et al. 2017, ApJL, 848, L23 Fruscione, A., McDowell, J. C., Allen, G. E., et al. 2006, Proc. SPIE, 6270, $62701 \mathrm{~V}$

Goldstein, A., Veres, P., Burns, E., et al. 2017, ApJL, 848, L14

Gottlieb, O., Nakar, E., \& Piran, T. 2018a, MNRAS, 473, 576

Gottlieb, O., Nakar, E., Piran, T., \& Hotokezaka, K. 2018b, MNRAS, 479, 588

Haggard, D., Nynka, M., Ruan, J. J., et al. 2017, ApJL, 848, L25

Hallinan, G., Corsi, A., Mooley, K. P., et al. 2017, Sci, 358, 1579
Hotokezaka, K., Kiuchi, K., Shibata, M., Nakar, E., \& Piran, T. 2018, arXiv: 1803.00599

Hunter, J. D. 2007, CSE, 9, 90

Kasen, D., Metzger, B., Barnes, J., Quataert, E., \& Ramirez-Ruiz, E. 2017, Natur, 551, 80

Kasliwal, M. M., Nakar, E., Singer, L. P., et al. 2017, Sci, 358, 1559

Kathirgamaraju, A., Barniol Duran, R., \& Giannios, D. 2018, MNRAS, 473, L121

Lamb, G. P., \& Kobayashi, S. 2017, MNRAS, 472, 4953

Lamb, G. P., Mandel, I., \& Resmi, L. 2018, arXiv:1806.03843

Lazzati, D., Deich, A., Morsony, B. J., \& Workman, J. C. 2017b, MNRAS, 471, 1652

Lazzati, D., Perna, R., Morsony, B. J., et al. 2017a, arXiv:1712.03237

Lyman, J. D., Lamb, G. P., Levan, A. J., et al. 2018, PhRvL, 120, 241103

Margutti, R., Alexander, K. D., Xie, X., et al. 2018, ApJL, 856, L18

Margutti, R., Berger, E., Fong, W., et al. 2017, ApJL, 848, L20

McCully, C., Hiramatsu, D., Howell, D. A., et al. 2017, ApJL, 848, L32

Mooley, K. P., Deller, A. T., Gottlieb, O., et al. 2018b, arXiv:1806.09693

Mooley, K. P., Nakar, E., Hotokezaka, K., et al. 2018a, Natur, 554, 207

Nakar, E., Gottlieb, O., Piran, T., Kasliwal, M. M., \& Hallinan, G. 2018, arXiv: 1803.07595

Nakar, E., \& Piran, T. 2017, ApJ, 834, 28

Nakar, E., \& Piran, T. 2018, MNRAS, 478, 407

Pian, E., DAvanzo, P., Benetti, S., et al. 2017, Natur, 551, 67

Ruan, J. J., Nynka, M., Haggard, D., Kalogera, V., \& Evans, P. 2018, ApJL, 853, L4

Savchenko, V., Ferrigno, C., Kuulkers, E., et al. 2017, ApJL, 848, L15

Schlafly, E. F., \& Finkbeiner, D. P. 2011, ApJ, 737, 103

Shappee, B. J., Simon, J. D., Drout, M. R., et al. 2017, Sci, 358, 1574

Smartt, S. J., Chen, T.-W., Jerkstrand, A., et al. 2017, Natur, 551, 75

Soares-Santos, M., Holz, D. E., Annis, J., et al. 2017, ApJL, 848, L16

Troja, E., Piro, L., Ryan, G., et al. 2018, MNRAS, 478, L18

Troja, E., Piro, L., van Eerten, H., et al. 2017, Natur, 551, 71

Valenti, S., Sand, D. J., Sheng, Y., et al. 2017, ApJL, 848, L24

van Eerten, H., Zhang, W., \& MacFadyen, A. 2010, ApJ, 722, 235

Verner, D. A., Ferland, G. J., Korista, K. T., \& Yakovlev, D. G. 1996, ApJ, 465,487

Wilms, J., Allen, A., \& McCray, R. 2000, ApJ, 542, 914

Xie, X., Zrake, J., \& MacFadyen, A. 2018, arXiv:1804.09345 